\title{
Intra-regular Left Almost Semigroups Characterized by Their Anti Fuzzy Ideals
}

\author{
Madad Khan \\ Department of Mathematics \\ COMSATS Institute of Information Technology \\ Abbottabad, Pakistan \\ E-mail: madadmath@yahoo.com \\ Tauseef Asif \\ Department of Mathematics \\ COMSATS Institute of Information Technology \\ Abbottabad, Pakistan \\ E-mail: tauseefmath@yahoo.com \\ Faisal \\ Department of Mathematics \\ COMSATS Institute of Information Technology \\ Abbottabad, Pakistan \\ E-mail: yousafzaimath@yahoo.com
}

\begin{abstract}
In this paper, we have introduced anti fuzzy quasi-ideals, anti fuzzy bi-(generalized bi-) ideals and anti fuzzy left (right, two-sided) ideals in LA-semigroup. Further we have characterized an intra-regular LA-semigroup by the properties of their anti fuzzy left (right, two-sided) ideals, anti fuzzy bi- (generalized bi-)ideals, anti fuzzy interior ideals and anti fuzzy quasi-ideals. Further we have shown that anti fuzzy two-sided ideals, anti fuzzy bi-ideals, anti fuzzy generalized bi-ideals, anti fuzzy interior ideals and anti fuzzy quasi-ideals coincide in an intra-regular LA-semigroup with left identity. Also we have proved that the set of anti fuzzy two-sided ideals of an intra-regular LA-semigroup $S$ with left identity forms a semilattice structure.
\end{abstract}

Keywords: LA-semigroup, Left invertive law, Medial law, Paramedial law, Anti fuzzy ideals

\section{Introduction}

Zadeh in 1965 has introduced fundamental concept of a fuzzy set which provides a natural frame-work for generalizing several basic notion of algebra. Kuroki $[1979,1981]$ has introduced fuzzy (left, right) ideals and bi-ideals in semigroups. Rosenfeld was the first who studied fuzzy sets in the structure of groups [Rosenfeld, 1971]. On the other hand, Biswas [Biswas, R, 1990] introduced the concept of anti fuzzy subgroups of groups. Hong and Jun [1998] defined anti fuzzy ideals in BCK-algebra. In [Akram, M., 2007], Akram and Dar worked on anti fuzzy left h-ideals in hemirings. In [Shabir, 2009] Shabir and Nawaz introduced the concept of anti fuzzy ideals in semigroups and characterized different classes of semigroups by the properties of their anti fuzzy ideals.

Our aim in this paper is to characterized an intra-regular LA-semigroup by the properties of their anti fuzzy left (right, two-sided) ideals, anti fuzzy (generalized) bi-ideals and anti fuzzy quasi-ideals.

A left almost semigroup (LA-semigroup) [Kazim, 1972] or Abel-Grassmann's groupoid (AG-groupoid) [Protić, P. V., 1995], is a groupoid $S$ holding left invertive law

$$
(a b) c=(c b) a, \text { for all } a, b, c \in S .
$$

In an LA-semigroup, the medial law [Kazim, 1972] holds,

$$
(a b)(c d)=(a c)(b d), \text { for all } a, b, c, d \in S .
$$

The left identity in an LA-semigroup if exists is unique [Mushtaq, 1978]. In an LA-semigroup $S$ with left identity the paramedial law holds,

$$
(a b)(c d)=(d b)(c a), \text { for all } a, b, c, d \in S .
$$


An LA-semigroup is a non-associative algebraic structure mid way between a groupoid and a commutative semigroup with wide applications in the theory of flocks. An LA-semigroup with right identity becomes a commutative semigroup with identity [Mushtaq, 1978]. If an LA-semigroup contains left identity, the following law holds,

$$
a(b c)=b(a c), \text { for all } a, b, c \in S \text {. }
$$

It is easy to note that if an LA-semigroup $S$ contains left identity then $e S=S e=S^{2}=S$. Some preliminaries are given below.

Let $S$ be an LA-semigroup. By LA-subsemigroup of $S$ we means a non-empty subset $A$ of $S$ such that $A^{2} \subseteq A$, and by a left (right) ideal of $S$ we mean a non-empty subset $A$ of $S$ such that $S A \subseteq A(A S \subseteq A)$. By two-sided ideals or simply ideal, we mean a non-empty subset of $S$ which is both a left and a right ideal of $S$. An LA-subsemigroup $A$ of $S$ is called bi-ideal of $S$ if $(A S) A \subseteq A$. A subset $A$ of $S$ is called generalized bi-ideal of $S$ if $(A S) A \subseteq A$. An LA-subsemigroup $A$ of $S$ is called interior ideal of $S$ if $(S A) S \subseteq A$.

A fuzzy subset $f$ of a given set $S$ is described as an arbitrary function $f: S \longrightarrow[0,1]$, where $[0,1]$ is the usual closed interval of real numbers. For any two fuzzy subsets $f$ and $g$ of $S, f \leq g$ means that, $f(x) \leq g(x)$ for all $x$ in $S$. The symbols $f \cap g$ and $f \cup g$ will means the following fuzzy subsets of $S$

$$
\begin{aligned}
& (f \cap g)(x)=\min \{f(x), g(x)\}=f(x) \wedge g(x) \\
& (f \cup g)(x)=\max \{f(x), g(x)\}=f(x) \vee g(x)
\end{aligned}
$$

for all $x$ in $S$.

Let $f$ and $g$ be any fuzzy subsets of an LA-semigroup $S$, then the product $f \circ g$ is defined by

$$
(f \circ g)(a)=\left\{\begin{array}{c}
\bigvee_{a=b c}\{f(b) \wedge g(c)\}, \text { if there exist } b, c \in S, \text { such that } a=b c \\
0, \\
\text { otherwise. }
\end{array}\right.
$$

Let $A$ be a subset of an LA-semigroup $S$, then the characteristic function of $A$, that is $C_{A}$ is defined by

$$
C_{A}(x)=\left\{\begin{array}{l}
1, \text { if } x \in A \\
0, \text { if } x \notin A
\end{array}\right.
$$

A fuzzy subset $f$ of an LA-semigroup $S$ is called a fuzzy LA-subsemigroup of $S$ if $f(x y) \geq f(x) \wedge f(y)$ for all $x, y \in S$.

A fuzzy subset $f$ of an LA-semigroup $S$ is called fuzzy left (right) ideal of $S$ if $f(x y) \geq f(y)(f(x y) \geq f(x))$ for all $x$, $y \in S$.

A fuzzy subset $f$ of an LA-semigroup $S$ is called fuzzy two-sided ideal of $S$ if it is both fuzzy left and fuzzy right ideal of $S$.

A fuzzy LA-subsemigroup $f$ of an LA-semigroup $S$ is called fuzzy bi-ideal of $S$ if $f((x y) z) \geq f(x) \wedge f(z)$, for all $x, y$ and $z \in S$.

A fuzzy subset $f$ of an LA-semigroup $S$ is called fuzzy generalized bi-ideal of $S$ if $f((x y) z) \geq f(x) \wedge f(z)$, for all $x, y$ and $z \in S$.

A fuzzy LA-subsemigroup $f$ of an LA-semigroup $S$ is called fuzzy interior ideal if $f((x a) y) \geq f(a)$, for all $x, a$ and $y \in S$.

A fuzzy subset $f$ of an LA-semigroup $S$ is called fuzzy quasi-ideal of $S$ if $(f \circ S) \cap(S \circ f) \subseteq f$.

Let $f$ and $g$ be any fuzzy subsets of an LA-semigroup $S$, then their anti product $f * g$ is defined by

$$
(f * g)(a)=\left\{\begin{array}{c}
\bigwedge_{a=b c}\{f(b) \vee g(c)\}, \text { if there exist } b, c \in S, \text { such that } a=b c \\
1, \\
\text { otherwise. }
\end{array}\right.
$$

Let $A$ be a subset of an LA-semigroup $S$, then the characteristic function of the complement of $A$, that is $C_{A^{c}}$ is defined by

$$
C_{A^{C}}(x)=\left\{\begin{array}{l}
0, \text { if } x \in A, \\
1, \text { if } x \notin A .
\end{array}\right.
$$

A fuzzy subset $f$ of an LA-semigroup $S$ is called anti fuzzy LA-subsemigroup of $S$ if $f(x y) \leq f(x) \vee f(y)$ for all $x, y \in S$. 
A fuzzy subset $f$ of an LA-semigroup $S$ is called anti fuzzy left (right) ideal of $S$ if $f(x y) \leq f(y)(f(x y) \leq f(x))$ for all $x$, $y \in S$.

A fuzzy subset $f$ of an LA-semigroup $S$ is called anti fuzzy two-sided ideal of $S$ if it is both anti fuzzy left ideal and anti fuzzy right ideal of $S$.

A fuzzy subset $f$ of an LA-semigroup $S$ is called anti fuzzy generalized bi-ideal of $S$ if $f((x y) z) \leq f(x) \vee f(z)$, for all $x, y$ and $z \in S$.

A fuzzy subset $f$ of an LA-semigroup $S$ is called anti fuzzy interior ideal of $S$ if $f((x a) y) \leq f(a)$, for all $x, a$ and $y \in S$.

A fuzzy subset $f$ of an LA-semigroup $S$ is called anti fuzzy quasi-ideal of $S$ if $(f * \Theta) \cup(\Theta * f) \supseteq f$, where $\Theta$ is the fuzzy subset of $S$ such that $\Theta(x)=0$, for all $x$ in $S$.

Example 1. Let $S=\{a, b, c\}$. The following multiplication table shows that $S$ is an LA-semigroup.

\begin{tabular}{c|ccc}
$*$ & $a$ & $b$ & $c$ \\
\hline$a$ & $a$ & $a$ & $a$ \\
$b$ & $a$ & $a$ & $c$ \\
$c$ & $a$ & $a$ & $a$
\end{tabular}

Define the fuzzy subset $f$ of $S$ as: $f(a)=0.4, f(b)=0.3$ and $f(c)=0.5$. Then clearly $f$ is an anti fuzzy LA-subsemigroup of $S$.

Define the fuzzy subset $f$ of $S$ as: $f(a)=0.2, f(b)=0.6$ and $f(c)=0.6$. Then clearly $f$ is an anti fuzzy two-sided ideal of $S$.

Lemma 1. Let $S$ be an LA-semigroup, then the following properties hold in $S$.

(i) $(f * g) * h=(h * g) * f$ for all fuzzy subsets $f, g$ and $h$ of $S$.

(ii) $(f * g) *(h * k)=(f * h) *(g * k)$ for all fuzzy subsets $f, g, h$ and $k$ of $S$.

Proof. It is simple.

Lemma 2. Let $S$ be an LA-semigroup with left identity, then the following properties hold in $S$.

(i) $f *(g * h)=g *(f * h)$ for all fuzzy subsets $f, g$ and $h$ of $S$.

(ii) $(f * g) *(h * k)=(k * h) *(g * f)$ for all fuzzy subsets $f, g, h$ and $k$ of $S$.

Proof. It is simple.

Let $F(S)$ denote the collection of all fuzzy subsets of an LA-semigroup $S$ with left identity, then $(F(S)$, *) becomes an LA-semigroup with left identity $\Theta$, that is $(F(S), *)$ satisfy all properties (1), (2), (3) and (4).

Lemma 3. Let $f$ be a fuzzy subset of an LA-semigroup $S$ with left identity, then $\Theta * f=f$, where $\Theta$ is the fuzzy subset of $S$ such that $\Theta(a)=0$, for all $a$ in $S$.

Proof. Let $f$ be any fuzzy subset of $S$ and $a$ be any element of $S$. Since $e$ is left identity in $S$ that is $a=e a$, for all $a$ in $S$, we have

$$
(\Theta * f)(a)=\bigwedge_{a=e a}\{\Theta(e) \vee f(a)\}=\bigwedge_{a=e a}\{0 \vee f(a)\}=\bigwedge_{a=e a} f(a)=f(a) .
$$

and so, $\Theta * f=f$.

Lemma 4. Let $f$ be a fuzzy subset of an LA-semigroup $S$. Then the following properties hold.

(i) $f$ is an anti fuzzy LA-subsemigroup of $S$ if and only if $f * f \supseteq f$.

(ii) $f$ is an anti fuzzy left (right) ideal of $S$ if and only if $\Theta * f \supseteq f(f * \Theta \supseteq f)$.

(iii) $f$ is an anti fuzzy two-sided ideal of $S$ if and only if $\Theta * f \supseteq f$ and $f * \Theta \supseteq f$.

Proof. It is simple. 
Lemma 5. Let A be a non-empty subset of an LA-semigroup $S$. Then A is a bi-ideal of $S$ if and only if $C_{A^{c}}$ is an anti fuzzy bi-ideal of $S$.

Proof. It is simple.

Lemma 6. Let $f$ be an anti fuzzy LA-subsemigroup of an LA-semigroup $S$. Then $f$ is an anti fuzzy bi-ideal of $S$ if and only if $(f * \Theta) * f \supseteq f$, where $\Theta$ is the fuzzy subset of $S$ such that $\Theta(x)=0$, for all $x$ in $S$.

Proof. It is simple.

Note that in an LA-semigroup $S$ with left identity, $\Theta$ can be considered as a fuzzy subset of $S$ such that $\Theta * \Theta=\Theta$ and $\Theta=C_{\Theta}$ for all $x \in \Theta$.

The principal left, right and two-sided ideals of an LA-semigroup $S$ generated by $a^{2}$ is denoted by $L\left[a^{2}\right], R\left[a^{2}\right]$ and $J\left[a^{2}\right]$. Note that the principal left, right and two-sided ideals generated by $a^{2}$ are equals, that is,

$$
L\left[a^{2}\right]=R\left[a^{2}\right]=J\left[a^{2}\right]=S a^{2}=a^{2} S=S a^{2} S=\left\{s a^{2}: s \in S\right\} .
$$

Lemma 7. Let A be a non-empty subset of an LA-semigroup S. Then the following properties holds.

(i) $A$ is an LA-subsemigroup if and only if $C_{A^{C}}$ is an anti fuzzy LA-subsemigroup of $S$.

(ii) $A$ is a left (right, two-sided) ideal of $S$ if and only if $C_{A^{C}}$ is an anti fuzzy left (right, two-sided) of $S$.

Proof. It is simple.

Lemma 8. Let $f$ be any anti fuzzy right ideal and $g$ be any anti fuzzy left ideals of $S$, then $f \cup g$ is an anti fuzzy quasi-ideal of $S$.

Proof. It is easy to observe the following,

$$
((f \cup g) * \Theta) \cup(\Theta *(f \cup g)) \supseteq(f * \Theta) \cup(\Theta * g) \supseteq f \cup g
$$

Lemma 9. Every anti fuzzy quasi-ideal of an LA-semigroup $S$ is an anti fuzzy LA-subsemigroup of $S$.

Proof. Let $f$ be any anti fuzzy quasi-ideal of $S$, then $f * f \supseteq f * \Theta$, and $f * f \supseteq \Theta * f$, therefore

$$
f * f \supseteq f * \Theta \cup \Theta * f \supseteq f .
$$

Hence $f$ is an anti fuzzy LA-subsemigroup of $S$.

A fuzzy subset $f$ of an LA-semigroup $S$ is called idempotent, if $f * f=f$, shortly $f^{2}=f$.

Lemma 10. In an LA-semigroup $S$, every idempotent anti fuzzy quasi-ideal of $S$ is an anti fuzzy bi-ideal of $S$.

Proof. Let $f$ be any anti fuzzy quasi-ideal of an LA-semigroup $S$, then by lemma $7, f$ is an anti fuzzy LA-subsemigroup an LA-semigroup $S$. Now by using (2) we have

$$
\begin{aligned}
&(f * \Theta) * f \supseteq(\Theta * \Theta) * f \supseteq \Theta * f \text { and } \\
&(f * \Theta) * f=(f * \Theta) *(f * f)=(f * f) *(\Theta * f) \\
& \supseteq \quad f *(\Theta * \Theta) \supseteq f * \Theta,
\end{aligned}
$$

which implies that $(f * \Theta) * f \supseteq(f * \Theta) \cup(\Theta * f) \supseteq f$.

Hence by lemma $6, f$ is an anti fuzzy bi-ideal of $S$.

Lemma 11. Let $f$ be an idempotent anti fuzzy quasi-ideal and $g$ be any anti fuzzy quasi ideal of an LA-semigroup $S$ with left identity, then $f * g$ or $g * f$ is an anti fuzzy bi-ideal of $S$. 
Proof. Clearly $f * g$ is an anti fuzzy LA-subsemigroup. Now using lemma 6, (1), (3) and (2), we have

$$
\begin{aligned}
((f * g) * \Theta) *(f * g) & =((\Theta * g) * f) *(f * g) \\
& \supseteq((\Theta * \Theta) * f) *(f * g) \\
& =(\Theta * f) *(f * g) \\
& =(g * f) *(f * \Theta) \\
& =((f * \Theta) * f) * g \supseteq(f * g) .
\end{aligned}
$$

Similarly we show that $g * f$ is an anti fuzzy bi-ideal of $S$.

Lemma 12. In an LA-semigroup $S$, each one sided anti fuzzy (left,right) ideal of $S$ is an anti fuzzy quasi-ideal of $S$.

Proof. It is obvious.

Corollary 1. In an LA-semigroup $S$, every anti fuzzy two sided ideal of $S$ is an anti fuzzy quasi-ideal of $S$.

Lemma 13. The product of two anti fuzzy left (right) ideals of an LA-semigroup $S$ with left identity is an anti fuzzy left (right) ideal of $S$.

Proof. Let $f$ and $g$ be any two anti fuzzy left ideals of $S$, then by using (4), we have

$$
\Theta *(f * g)=f *(\Theta * g) \supseteq f * g .
$$

Let $f$ and $g$ be any two anti fuzzy right ideals of $S$, then by using (2), we have

$$
(f * g) * \Theta=(f * g) *(\Theta * \Theta)=(f * \Theta) *(g * \Theta) \supseteq f * g .
$$

Lemma 14. If $g$ is an anti fuzzy right ideal of an LA-semigroup $S$ with left identity, then $g * \Theta=g$.

Proof. Let $a \in S$, then by using (1), we have $a=e a=(e e) a=a$. Thus

$$
\begin{aligned}
(g * \Theta)(a) & =\bigwedge_{a=(a e) e}\{g(a e) \vee \Theta(e)\} \leq\{g(a e) \vee \Theta(e)\} \\
& \leq\{g(a) \vee 0\}=g(a)
\end{aligned}
$$

Lemma 15. In an LA-semigroup $S$, each one sided anti fuzzy (left,right) ideal of $S$ is an anti fuzzy generalized bi-ideal of $S$.

Proof. Assume that $f$ be any anti fuzzy left ideal of $S$ and $a, b, c \in S$, then by using (1) we have, $f((a b) c)=f((c b) a) \leq$ $f(a)$ and $f((a b) c) \leq f(c)$. Thus $f((a b) c) \leq f(a) \vee f(c)$. Similarly in case of right ideal.

An element $a$ of an LA-semigroup $S$ is called an intra-regular if there exist $x, y \in S$ such that $a=\left(x a^{2}\right) y$ and $S$ is called an intra-regular if every element of $S$ is intra-regular.

Example 2. Let $S=\{1,2,3,4,5,6\}$ be an LA-semigroup with left identity 5 with the following multiplication table.

\begin{tabular}{l|llllll}
$\cdot$ & 1 & 2 & 3 & 4 & 5 & 6 \\
\hline 1 & 1 & 2 & 3 & 4 & 5 & 6 \\
2 & 4 & 1 & 5 & 2 & 6 & 3 \\
3 & 5 & 3 & 1 & 6 & 4 & 2 \\
4 & 2 & 4 & 6 & 1 & 3 & 5 \\
5 & 3 & 6 & 2 & 5 & 1 & 4 \\
6 & 6 & 5 & 4 & 3 & 2 & 1
\end{tabular}

Clearly $S$ is an intra-regular because $\left(2 \cdot 1^{2}\right) \cdot 4=1,\left(4 \cdot 2^{2}\right) \cdot 4=2,\left(5 \cdot 3^{2}\right) \cdot 2=3,\left(6 \cdot 4^{2}\right) \cdot 3=4,\left(4 \cdot 5^{2}\right) \cdot 3=5,\left(3 \cdot 6^{2}\right) \cdot 2$. 
Lemma 16. A fuzzy subset $f$ of an intra-regular LA-semigroup $S$ is an anti fuzzy right ideal if and only if it is an anti fuzzy left ideal.

Proof. Assume that $f$ is an anti fuzzy right ideal of $S$. Since $S$ is intra-regular, so for each $a \in S$ there exist $x, y \in S$ such that $a=\left(x a^{2}\right) y$. So by using (1), we have

$$
f(a b)=f\left(\left(\left(x a^{2}\right) y\right) b\right)=f\left((b y)\left(x a^{2}\right)\right) \leq f(b y) \leq f(b) .
$$

Conversely, assume that $f$ is an anti fuzzy left ideal of $S$, then using (1), we have

$$
f(a b)=f\left(\left(\left(x a^{2}\right) y\right) b\right)=f\left((b y)\left(x a^{2}\right)\right) \leq f\left(x a^{2}\right) \leq f\left(a^{2}\right) \leq f(a) .
$$

Lemma 17. Every anti fuzzy two-sided ideal of an intra-regular LA-semigroup $S$ with left identity is idempotent.

Proof. Assume that $f$ is an anti fuzzy two-sided ideal of $S$, then clearly $f * f \supseteq f * \Theta \supseteq f$. Since $S$ is an intra-regular LA-semigroup, so for each $a \in S$ there exist $x, y \in S$ such that $a=\left(x a^{2}\right) y$, so by using (4) and (1) we have

$$
a=\left(x a^{2}\right) y=(x(a a)) y=(a(x a)) y=(y(x a)) a .
$$

Thus, we have

$$
\begin{aligned}
(f * f)(a) & =\bigwedge_{a=(y(x a)) a}\{f(y(x a)) \vee f(a)\} \leq f(y(x a)) \vee f(a) \\
& \leq f(a) \vee f(a)=f(a) .
\end{aligned}
$$

Hence $f * f=f$.

Theorem 1. For a fuzzy subset $f$ of an intra-regular LA-semigroup $S$ with left identity, the following conditions are equivalent.

(i) $f$ is an anti fuzzy bi-ideal of $S$.

(ii) $f$ is an anti fuzzy generalized bi-ideal of $S$.

Proof. (i) $\Longrightarrow($ ii $)$

Let $f$ be any anti fuzzy bi-ideal of $S$, then obviously $f$ is an anti fuzzy generalized bi-ideal of $S$.

Now $(i i) \Rightarrow(i)$

Let $f$ be any anti fuzzy generalized bi-ideal of $S$, and $a, b \in S$. Then, since $S$ is an intra-regular LA-semigroup, so for each $a \in S$ there exist $x, y \in S$ such that $a=\left(x a^{2}\right) y$. So by using (3), (2) and (4), we have

$$
\begin{aligned}
f(a b) & =f\left(\left(\left(x a^{2}\right) y\right) b\right)=f\left(\left(\left(x a^{2}\right)(e y)\right) b\right)=f\left(\left((y e)\left(a^{2} x\right)\right) b\right) \\
& =f\left(\left(a^{2}((y e) x)\right) b\right)=f(((a a)((y e) x)) b) \\
& =f(((x(y e))(a a)) b)=f((a((x(y e)) a)) b) \leq f(a) \vee f(b) .
\end{aligned}
$$

Therefore $f$ is an anti fuzzy bi-ideal of $S$.

Theorem 2. For a fuzzy subset $f$ of an intra-regular LA-semigroup $S$ with left identity, the following conditions are equivalent.

(i) $f$ is an anti fuzzy two sided ideal of $S$.

(ii) $f$ is an anti fuzzy bi-ideal of $S$.

Proof. $($ i $) \Longrightarrow($ ii $)$

Let $f$ be any anti fuzzy two sided ideal of $S$, then obviously $f$ is an anti fuzzy bi-ideal of $S$.

Now $(i i) \Rightarrow(i)$ 
Let $f$ be any anti fuzzy bi-ideal of $S$ and for any $a, b \in S$. Since $S$ is intra-regular, so there exists $x, y$ and $u, v \in S$ such that $a=\left(x a^{2}\right) y$ and $b=\left(u b^{2}\right) v$. Therefore by using (1), (3), (2) and (4), we have

$$
\begin{aligned}
f(a b) & =f\left(\left(\left(x a^{2}\right) y\right) b\right)=f\left((b y)\left(x a^{2}\right)\right)=f\left(\left(a^{2} x\right)(y b)\right)=f\left(((y b) x) a^{2}\right) \\
& =f(((y b) x)(a a))=f((a a)(x(y b)))=f(((x(y b)) a) a) \\
& =f\left(\left((x(y b))\left(\left(x a^{2}\right) y\right)\right) a\right)=f\left(\left(\left(x a^{2}\right)((x(y b)) y)\right) a\right) \\
& =f\left(\left((y(x(y b)))\left(a^{2} x\right)\right) a\right)=f\left(\left(a^{2}((y(x(y b))) x)\right) a\right) \\
& =f(((a a)((y(x(y b))) x)) a)=f(((x(y(x(y b))))(a a)) a) \\
& =f((a((x(y(x(y b)))) a)) a) \leq f(a) \vee f(a)=f(a) . \text { And } \\
f(a b) & =f\left(a\left(\left(u b^{2}\right) v\right)\right)=f\left(\left(u b^{2}\right)(a v)\right)=f\left((v a)\left(b^{2} u\right)\right) \\
& =f\left(b^{2}((v a) u)\right)=f((b b)((v a) u))=f((((v a) u) b) b) \\
& =f\left(\left(((v a) u)\left(\left(u b^{2}\right) v\right)\right) b\right)=f\left(\left(\left(u b^{2}\right)(((v a) u) v)\right) b\right) \\
& =f\left(\left((v((v a) u))\left(b^{2} u\right)\right) b\right)=f\left(\left(b^{2}((v((v a) u)) u)\right) b\right) \\
& =f(((b b)((v((v a) u)) u)) b)=f(((u(v((v a) u)))(b b)) b) \\
& =f((b((u(v((v a) u))) b)) b) \leq f(b) \vee f(b)=f(b)
\end{aligned}
$$

Corollary 2. An anti fuzzy right ideal of an LA-semigroup $S$ is an anti fuzzy bi-ideal of $S$.

Theorem 3. For a fuzzy subset $f$ of an intra-regular LA-semigroup $S$ with left identity, the following conditions are equivalent.

(i) $f$ is an anti fuzzy two sided ideal of $S$.

(ii) $f$ is an anti fuzzy interior ideal of $S$.

Proof. $(i) \Rightarrow($ ii $)$

Let $f$ be any anti fuzzy two sided ideal of $S$, then obviously $f$ is an anti fuzzy interior ideal of $S$.

Now $(i i) \Rightarrow(i)$

Let $f$ be any anti fuzzy interior ideal of $S$ and $a, b \in S$. Since $S$ is intra-regular LA-semigroup, so there exist $x, y$ and $u, v \in S$ such that $a=\left(x a^{2}\right) y$ and $b=\left(u b^{2}\right) v$, thus by using (1), (4) and (2), we have

$$
\begin{aligned}
f(a b) & \left.=f\left(\left(x a^{2}\right) y\right) b\right)=f\left((b y)\left(x a^{2}\right)\right)=f((b y)(x(a a))) \\
& =f((b y)(a(x a)))=f((b a)(y(x a))) \leq f(a) .
\end{aligned}
$$

Also by using (4), (3) and (2) we have

$$
\begin{aligned}
f(a b) & =f\left(a\left(\left(u b^{2}\right) v\right)\right)=f\left(\left(u b^{2}\right)(a v)\right)=f((b(u b))(a v)) \\
& =f((v a)((u b) b))=f((u b)((v a) b)) \leq f(b) .
\end{aligned}
$$

Hence $f$ is a fuzzy two sided ideal of $S$.

Corollary 3. An anti fuzzy right ideal of an LA-semigroup $S$ with left identity is an anti fuzzy interior ideal of $S$.

Theorem 4. A fuzzy subset $f$ of an intra-regular LA-semigroup $S$ with left identity is an anti fuzzy two-sided ideal of $S$ if and only if it is an anti fuzzy quasi-ideal of $S$.

Proof. Let $f$ be any anti fuzzy two-sided ideal of $S$, then obviously $f$ is an anti fuzzy quasi-ideal of $S$.

Conversely, assume that $f$ is an anti fuzzy quasi-ideal of $S$. Let $a$ be an arbitrary element of $S$. Then, since $S$ is intraregular, so there exist elements $x$ and $y$ in $S$ such that $a=\left(x a^{2}\right) y$, by using (2) and (3), we have

$$
\begin{aligned}
a & =\left(x a^{2}\right) y=\left(x a^{2}\right)(e y)=(x e)\left(a^{2} y\right)=a^{2}((x e) y)=(a a)((x e) y) \\
& =(y a)((x e) a)=(y(x e))(a a)=a((y(x e)) a) .
\end{aligned}
$$


Thus, we have

$$
\begin{aligned}
(f * \Theta)(a) & =\bigwedge_{a=a((y(x e)) a)}\{f(a) \vee \Theta((y(x e)) a)\} \leq f(a) \vee \Theta((y(x e)) a) \\
& \leq f(a) \vee 0=f(a),
\end{aligned}
$$

which implies that $f * \Theta \subseteq f$, so by using (2), we have

$$
\begin{aligned}
f * \Theta & =(\Theta * f) *(\Theta * \Theta)=(\Theta * \Theta) *(f * \Theta) \\
& =\Theta *(f * \Theta) \subseteq \Theta * f,
\end{aligned}
$$

which implies that $\Theta * f \supseteq f * \Theta$. Therefore

$$
\Theta * f \supseteq(f * \Theta) \cup(\Theta * f) \supseteq f,
$$

which shows that $f$ is an anti fuzzy left ideal of $S$ and by lemma 17, $f$ is an anti fuzzy right ideal of $S$. Hence $f$ is an anti fuzzy two-sided ideal of $S$.

Theorems 24, 19, 21 and 22 shows that anti fuzzy two-sided ideals, anti fuzzy bi-ideals, anti fuzzy generalized bi-ideals, anti fuzzy interior ideals and anti fuzzy quasi-ideals coincide in an intra-regular LA-semigroup with left identity.

Lemma 18. Let $A$ and $B$ be any non-empty subsets of an LA-semigroup $S$, then the following properties hold.

(i) $C_{A^{C}} \cup C_{B^{C}}=C_{A^{C} \cup B^{C}}$.

(ii) $C_{A^{C}} * C_{B^{C}}=C_{A^{C} B^{C}}$.

Proof. It is straight forward.

A subset $A$ of an LA-semigroup $S$ is called semiprime if, $a^{2} \in A$ implies $a \in A$ for all $a$ in $S$.

A fuzzy subset $f$ of an LA-semigroup $S$ is called anti fuzzy semiprime if, $f(a) \leq f\left(a^{2}\right)$ for all $a$ in $S$.

Theorem 5. A non-empty subset A of an LA-semigroup $S$ is semiprime if and only if the characteristic function of the complement of $A$, that is $C_{A^{C}}$ is anti fuzzy semiprime.

Proof. Let $a^{2} \in A$, since $A$ is semiprime so $a \in A$, hence $C_{A^{c}}(a)=0=C_{A^{c}}\left(a^{2}\right)$. Also if $a^{2} \notin A$, then

$$
C_{A^{c}}(a) \leq 1=C_{A^{c}}\left(a^{2}\right) \text {. }
$$

In both cases, we have $C_{A^{c}}(a) \leq C_{A^{C}}\left(a^{2}\right)$ for all $a \in S$, which implies that $C_{A^{c}}$ is an anti fuzzy semiprime.

Conversely, assume that $a^{2} \in A$, since $C_{A^{c}}$ is an anti fuzzy semiprime, so we have

$$
C_{A^{c}}(a) \leq C_{A^{c}}\left(a^{2}\right)=0,
$$

which implies that $a \in A$. Hence $A$ is semiprime.

Lemma 19. In an intra-regular LA-semigroup $S$, every anti fuzzy interior ideal is an anti fuzzy semiprime.

Proof. Let $f$ be an anti fuzzy interior ideal of $S$ and $a$ be any element of S. Since $S$ is an intra-regular LA-semigroup, so there exist $x, y \in S$ such that $a=\left(x a^{2}\right) y$, so we have

$$
f(a)=f\left(\left(x a^{2}\right) y\right) \leq f\left(a^{2}\right) .
$$

Hence $f$ is an anti fuzzy semiprime.

Theorem 6. For an LA-semigroup $S$ with left identity, the following conditions are equivalent.

(i) $S$ is intra-regular.

(ii) $R \cap L \supseteq R L$, for every right ideal $R$ and every left ideal $L$ of $S$ and right ideal $R$ is semiprime. 
Proof. $(i) \Longrightarrow(i i)$ : It is obvious.

(ii) $\Longrightarrow($ i $)$

Let $a \in S$, then clearly $a \in S a$, where $S a$ is a left ideal of $S$ and $a^{2} \in a^{2} S$, where $a^{2} S$ is a right ideal of $S$. By assumption (ii) right ideal of $S$ is semiprime, which implies that $a \in a^{2} S$, thus by using (2) we have

$$
\begin{aligned}
a & \in a^{2} S \cap S a \subseteq\left(a^{2} S\right)(S a)=(a S)\left(S a^{2}\right)=(a S)((S S)(a a)) \\
& =(a S)((a a)(S S)) \subseteq(S S)\left(a^{2} S\right)=\left(S a^{2}\right)(S S)=\left(S a^{2}\right) S .
\end{aligned}
$$

Therefore there exist $x, y \in S$ such that $a=\left(x a^{2}\right) y$. Hence $S$ is intra-regular.

Theorem 7. In an LA-semigroup $S$ with left identity the following conditions are equivalent.

(i) $S$ is intra-regular.

(ii) $R \cap L=R L$ for every right ideal $R$ and every left ideal $L$ of $S$ and right ideal $R$ is semiprime.

Proof. It is available in [Shabir, M., 2009].

Theorem 8. If $S$ is an intra-regular LA-semigroup with left identity then, $f \cup g=f * g$ for every anti fuzzy left ideal $f$ and every anti fuzzy right ideal $g$ of $S$ and anti fuzzy right ideal $g$ is anti fuzzy semiprime.

Proof. Assume that $S$ is an intra-regular LA-semigroup. Let $f$ be any anti fuzzy left ideal and $g$ be any anti fuzzy right ideal of $S$, then we have

$$
f * g \supseteq \Theta * f \supseteq f \text { and } f * g \supseteq g * \Theta \supseteq g \text { which implies that } f * g \supseteq f \cup g \text {. }
$$

Since $S$ is an intra-regular LA-semigroup, so for each $a \in S$ there exist $x, y \in S$ such that $a=\left(x a^{2}\right) y$, now by using (4) and (1) we have

$$
a=\left(x a^{2}\right) y=(x(a a)) y=(a(x a)) y=(y(x a)) a .
$$

Therefore, we have

$$
\begin{aligned}
(f * g)(a) & =\bigwedge_{a=(y(x a)) a}\{f(y(x a)) \vee g(a)\} \\
& \leq f(a) \vee g(a)=(f \cup g)(a) .
\end{aligned}
$$

Thus $f * g=f \cup g$. Now by using (3), (2) and (4) we have

$$
\begin{aligned}
g(a) & =g\left(\left(x a^{2}\right) y\right)=g\left(\left(x a^{2}\right)(e y)\right)=g\left((y e)\left(a^{2} x\right)\right) \\
& =g\left(a^{2}((y e)(x))\right) \leq g\left(a^{2}\right) .
\end{aligned}
$$

Hence $g$ is anti fuzzy semiprime.

Lemma 20. If $f$ and $g$ are any anti fuzzy two-sided ideals of an intra-regular LA-semigroup $S$ with left identity, then $f * g=f \cup g$.

Proof. Let $f$ and $g$ be any anti fuzzy two-sided ideals of $S$, then obviously $f * g \supseteq f \cup g$. Since $S$ is an intra-regular LA-semigroup so for each element $a$ in $S$ there exist elements $x$ and $y$ in $S$ such that $a=\left(x a^{2}\right) y$, now by using (4) and (1) we have

$$
a=\left(x a^{2}\right) y=(x(a a)) y=(a(x a)) y=(y(x a)) a .
$$

Therefore, we have

$$
\begin{aligned}
(f * g)(a) & =\bigwedge_{a=(y(x a)) a}\{f(y(x a)) \vee g(a)\} \leq f(y(x a)) \vee g(a) \\
& \leq f(a) \vee g(a)=(f \cup g)(a) .
\end{aligned}
$$

which implies that $f * g \subseteq f \cup g$. Hence $f * g=f \cup g$.

Theorem 9. If $S$ is an intra-regular LA-semigroup with left identity, then, $f \cup g \supseteq(f * g) * f$, for every fuzzy right ideal $f$ and every fuzzy generalized bi-ideal (fuzzy quasi-ideal) $g$ of $S$ and fuzzy right ideal $f$ is fuzzy semiprime. 
Proof. Assume that $S$ is an intra-regular LA-semigroup. Let $f$ and $g$ be any anti fuzzy right and anti fuzzy generalized bi-ideal (fuzzy quasi-ideal) of $S$. Since $S$ is an intra-regular, so for each $a \in S$ there exist $x, y \in S$ such that $a=\left(x a^{2}\right) y$, then by using (4), (1), (3) and (2) we have

$$
\begin{aligned}
a & =\left(x a^{2}\right) y=(a(x a)) y=(y(x a)) a=\left(y\left(x\left(\left(x a^{2}\right) y\right)\right)\right) a=\left(y\left(\left(x a^{2}\right)(x y)\right)\right) a \\
& =\left(\left(x a^{2}\right)(y(x y))\right) a=((x(a a))(y(x y))) a=((a(x a))(y(x y))) a \\
& =(((y(x y))(x a)) a) a=(((a x)((x y) y)) a) a .
\end{aligned}
$$

Thus we have

$$
\begin{aligned}
((f * g) * f)(a) & =\bigwedge_{a=(((a x)((x y) y)) a) a}\{(f((a x)((x y) y)) \vee g(a)) \vee f(a)\} \\
& \leq(f((a x)((x y) y)) \vee g(a)) \vee f(a) \\
& \leq(f(a) \vee g(a)) \vee f(a)=(f \cup g)(a) .
\end{aligned}
$$

Therefore $f \cup g \supseteq(f * g) * f$, and by using (3), (2)and (4) we have

$$
\begin{aligned}
f(a) & =f\left(\left(x a^{2}\right) y\right)=f\left(\left(x a^{2}\right)(e y)\right)=f\left((y e)\left(a^{2} x\right)\right) \\
& =f\left(a^{2}((y e)(x))\right) \leq f\left(a^{2}\right) .
\end{aligned}
$$

Hence $g$ is fuzzy semiprime.

Theorem 10. The set of anti fuzzy two-sided ideals of an intra-regular LA-semigroup $S$ with left identity forms a semilattice structure with identity $\Theta$.

Proof. Let $\mathbb{I}_{\Theta}$ be the set of anti fuzzy ideals of an intra-regular LA-semigroup $S$ and $f, g$ and $h \in \mathbb{I}_{\Theta}$, then by lemma [14], $\mathbb{I}_{\Theta}$ is closed and by lemmas [18] and [31], we have $f=f^{2}$ and $f * g=f \cup g$, where $f$ and $g$ are anti fuzzy ideals of $S$. Clearly $f * g=g * f$, and now by using (1), we get $(f * g) * h=(h * g) * f=f *(g * h)$. Also by using (1) and lemma [3], we have $f * \Theta=(f * f) * \Theta=(\Theta * f) * f=f * f=f$.

Note that in an intra-regular LA-semigroup $S$ with left identity every anti fuzzy two-sided ideal becomes anti fuzzy semiprime.

An anti fuzzy two-sided ideal $f$ of an LA-semigroup $S$ is said to be strongly irreducible if and only if for any anti fuzzy two-sided ideals $g$ and $h$ of $S, g \cup h \supseteq f$ implies that $g \supseteq f$ or $h \supseteq f$.

The set of anti fuzzy two-sided ideals of an LA-semigroup $S$ is called totally ordered under inclusion if for any anti fuzzy two-sided ideals $f$ and $g$ of $S$ either $f \supseteq g$ or $g \supseteq f$.

An anti fuzzy two-sided ideal $h$ of an LA-semigroup $S$ is called anti fuzzy prime ideal of $S$, if for any anti fuzzy two-sided ideals $f$ and $g$ of $S, f * g \supseteq h$, implies that $f \supseteq h$ or $g \supseteq h$.

Theorem 11. In an intra-regular LA-semigroup $S$ with left identity, an anti fuzzy two-sided ideal is strongly irreducible if and only if it is anti fuzzy prime.

Proof. It follows from lemmas 18 and 31.

Theorem 12. Every fuzzy two-sided ideal of an intra-regular LA-semigroup $S$ with left identity is fuzzy prime if and only if the set of fuzzy two-sided ideals of $S$ is totally ordered under inclusion.

Proof. It follows from lemmas 18 and 31.

\section{References}

Akram, M. and Dar, K. H. (2007). On anti fuzzy left h-ideals in hemirings. International Mathematical Forum, 46, $2295-2304$.

Asif, T. (2010). On Fuzzification in left almost semigroups, MS. Thesis, CIIT, Abbottabad.

Biswas, R. (1990). Fuzzy subgroups and anti fuzzy subgroups. Fuzzy sets and systems, 35, 121 - 124.

Hong, S. M. and Jun, Y. B. (1998). Anti fuzzy ideals in BCK-algebra. Kyungpook Math. J., 38, 145 - 150.

Ježek, J. and Kepka, T. (1993). A note on medial division groupoids. Proc. Amer. Math. Soc., 2, 119, 423 - 426. 
Ježek, J. and Kepka, T. (2000). The equational theory of paramedial cancellation groupoids. Czech. Math. J., 50, 125, 2534.

Kazim, M. A. and Naseeruddin, M. (1972). On almost semigroups. The Alig. Bull. Math., 2, 1 - 7.

Kuroki, N. (1979). Fuzzy bi-ideals in semigroups. Comment. Math. Univ. St. Pauli, 27, $17-21$.

Kuroki, N. (1981). On fuzzy ideals and bi-ideals in semigroups. Fuzzy Sets and Systems, 5, 203 - 215.

Kuroki, N. (1982). On fuzzy semiprime ideals in semigroups. Fuzzy sets and Systems, 8, 71 - 79.

Kuroki, N. (1991). On fuzzy semigroups. Inform. Sci., 53, 203 - 236.

Kuroki, N. (1992). Fuzzy generalized bi-ideals in semigroups. Inform. Sci., 66, 235 - 243.

Mordeson, J. N., Malik, D. S. and Kuroki, N. (2003). Fuzzy semigroups. Springer-Verlag, Berlin, Germany.

Mushtaq, Q. and Yusuf, S. M. (1978). On LA-semigroups. The Alig. Bull. Math., 8, 65 - 70.

Protić, P. V. and Stevanović, N. (1995). AG-test and some general properties of Abel-Grassmann's groupoids. PU. M. A., 4, 6, $371-383$.

Rosenfeld, A. (1971). Fuzzy groups. J. Math. Anal. Appl., 35, 512 - 517.

Shabir, M. and Nawaz, Y. (2009). Semigroups characterized by the properties of their anti fuzzy ideals. Journal of advanced Research in Pure Mathematics, 3, 42 - 59.

Zadeh, L. A. (1965). Fuzzy sets. Inform. Control, 8, 338 - 353. 